\title{
Orthogonal Extraction Ion Mobility Spectrometry
}

\author{
Victor V. Laiko \\ MassTech, Inc., Columbia, Maryland, USA
}

Ion Mobility Spectrometry is a powerful tool for the study of molecular conformations, separation of mass isomers, and analysis of complex mixtures and suppression of chemical background. The factors that limit the capabilities of the technique include its relatively low resolving power and duty cycle. New principle of gas-phase ion separation, based on ion focusing under the influence of electrostatic field and stationary in time gas flow, is proposed. Both analytical calculations and a numerical simulation show that a diffusion-limited resolution of several hundred can be achieved. The new type of ion mobility analyzer is called orthogonal extraction IMS. The proposed ortho-IMS can be interfaced with commercial mass spectrometers and offers the theoretical resolution of several hundred and ion transmission close to 100\%. (J Am Soc Mass Spectrom 2006, 17, 500-507) (c) 2006 American Society for Mass Spectrometry

$\mathrm{T}$ The mobility of a gas-phase ion is a measure of how rapidly it moves through a buffer gas under the influence of an electrical field. The mobility of a polyatomic ion depends on its average collision cross section, which in turn depends on its geometry. The measurements of ion mobility form the basis of sensitive and selective analytical technique, ion mobility spectrometry [1, 2]. Developed by Cohen and Karasek in 1970 [1], IMS has been used for the detection of drugs, chemical warfare agents, explosives, and environmental pollutants [3].

Although IMS is best known for security applications [4], its use in analytical biochemistry is on the rise, especially with the invention of ESI [5] and MALDI [6, 7], the two "soft" ionization techniques that enable large intact biomolecular ions to be generated in the gasphase. Historically, electrospray ionization was first invented and interfaced with IMS by Dole and coworkers in 1972 [8], long before MS application of ESI source was demonstrated by Fenn et al. [5] at the end of the 1980s. In the years 1989-1994, efforts of several groups were directed to improving the performance of ESI-IMS instruments [9-11]. Still, the interpretation of the recorded ion mobility spectra was possible only after comparison with ESI-MS [11]. At the end of the 1990s, IMS instruments had been coupled with different mass analyzers, including quadrupoles, ion traps, time-offlight (TOF), and FT-ICR MS. These hybrid instruments have enabled rapid separation of complex biological mixtures (tryptic digests and environmental samples) and have contributed to conformational information for biological analytes [12]. In recent years, (2002-2005), a

Published online February 28, 2006

Address reprint requests to Dr. V. V. Laiko, MassTech, Inc., 6259 Columbia Gateway Drive, Columbia, MD 21046, USA. E-mail: vlaiko@apmaldi.com new generation of ESI-IMS-MS and MALDI-IMS-MS have been developed and successfully applied for the numerous biochemical problems, including the study of noncovalent complex formation/structure $[13,14]$; the study of post-translational protein modifications [15]; the decreasing of the complexity of MS spectra of peptide mixtures [16, 17]; and the investigation of biomolecular cluster formation/structure [18, 19]. From this incomplete list of successful application of IMS-MS, clearly, incorporation of an ion mobility separation stage between an ion source and mass spectrometer provides a unique tool for the study of molecular conformations, for the separation of isomers, for enhanced analysis of very complex mixtures, and for the separation of analyte signal from the chemical noise background.

Surprisingly, more then three decades of the development of IMS has not brought a big variety of instrument types. Today, as thirty years ago, the drift-tube ion mobility spectrometer (DT-IMS) is still the prevailing type of ion mobility instrument. The operational principle of DT-IMS is similar to that of the time-of-flight (TOF) mass spectrometer invented at the end of 19th century by Tompson. In TOF instruments, ions with a certain kinetic energy are gated in a field-free drift tube. In the vacuum of the drift tube chamber, ions move with constant velocities proportional to the square root of ion energies. At the end of the tube, time-of-flights of the ions are measured by a detector. Knowing the time-of-flights, the masses of ions can be easily calculated. In DT-IMS, ions produced by the ion source are also gated by the inlet grid into a drift tube. The major difference between DT-IMS and TOF is that the drift tube of IMS is not evacuated but is filled with a buffer gas at relatively high-pressure. Under high-pressure conditions, ion velocities in the drift tube are propor- 
tional to the applied electrical field. If a uniform electrical field is maintained inside the drift tube, ion velocities are proportional to the respective ion mobilities. By measuring ion time-of-flights through the drift tube using a suitable detector, ion mobility spectrum is recorded.

Similar in design, TOF MS and DT-IMS share the same advantages and disadvantages. The design simplicity and speed of spectra recording are obviously a plus. Low resolution and low duty cycle constitute essential problems for both instruments. For a long time, TOF instruments were treated as lowperformance devices. The revolutionary inventions of ion reflector by Mamyrin et al. [20] and orthogonal acceleration TOF by Dodonov et al. [21, 22] have provided the bases for the increase in resolution of modern TOF MS from few hundred to several tens of thousand without compromising the sensitivity. Nothing like these have happened in IMS yet.

The resolution of the best modern DT-IMS instruments is still less then 100-almost at the same level as 30 years ago [4]. The fundamental factor that limits the resolution is the gas-phase diffusion broadening of ion packet during its propagation through the drift tube [23]. This phenomenon has already been investigated in detail [23]. The diffusion-limited resolution $R_{D}$ does not depend on the instrument geometry, ion mobility etc. For singly-charged ions, $R_{D}$ can be calculated [23] as $R_{D}$ $=16.5(U / T)^{1 / 2}$, where $U$ is the voltage applied across of the drift tube and $T$ is the buffer gas absolute temperature. If the temperature $T$ is $\approx 300^{\circ} \mathrm{K}$ (typically even higher temperatures are used to avoid various undesirable phenomena, like cross-contamination and cluster formation), there is only one way to increase the resolution limit of DT-IMS - that is to increase the applied voltage $U$. Simple calculation shows that to reach the resolution of 300 the voltages of $100 \mathrm{kV}$ or higher should be used.

The finite duration of injection pulses is another important factor that affects both the resolution and sensitivity of DT-IMS [23]. The shorter the injection period (when the gate grid is opened) used, the lower are the instrument duty cycle and sensitivity. To make better use of generated ion current, Fourier and, recently, Hadamard transform signal detection schemes were suggested [24, 25]. Some improvement in signalto-noise was reported in both cases, but practical value of both techniques is yet to be proven.

Another type of the device for the separation of charged particles, based on their mobilities, is known as a differential mobility analyzer (DMA). DMAs are commonly used for separation/detection of aerosol particles. In 1996, a successful application of DMA for analysis of electrospray-generated biomolecules was reported [26]. DMA is a filter-like device that is designed to transmit ions only in a narrow mobility window. A continuous stream of ions is injected into the drift region, confined by the two metal plates, through the inlet slit. The voltage applied to the plate

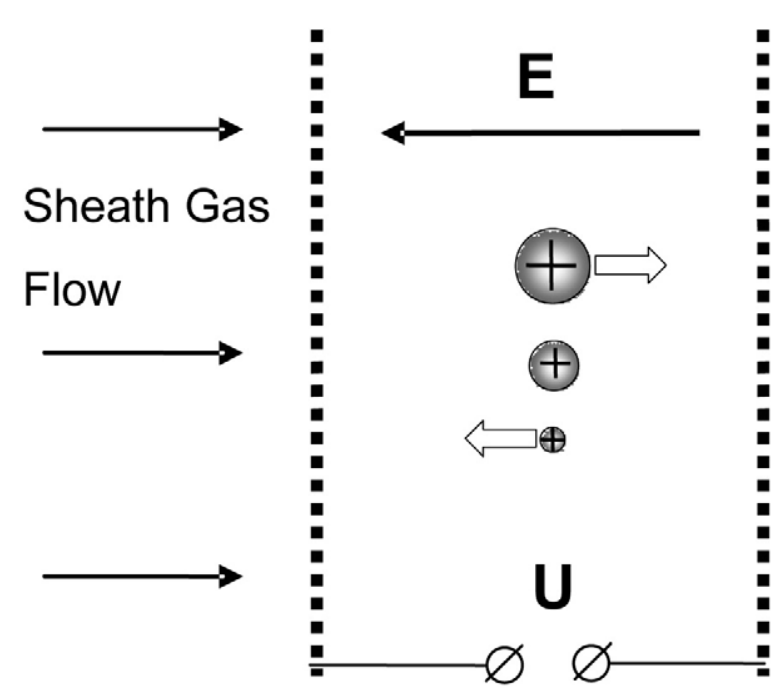

Figure 1. Parallel flow mobility analyzer of J. Zeleny.

produces the electrostatic field. The flow of sheath gas is maintained in the drift region. Only ions with a certain range of mobility exit the device through the outlet slit in the opposite metal plate. The major factor limiting the resolution of DMA is, again, ion random diffusion in a drift region [27]. Like for DT-IMS, diffusion-limited resolution of DMA [27] depends mostly on the square root of applied voltage. For several reasons [27], the resolution practically achievable in a DMA apparatus is even lower then that for DT-IMS. For example, recently DMA was interfaced with electrospray source on one side and with TOF mass spectrometer on another side [28]. The DMA resolution of only 20-25 was reported [28].

To complete the brief review of existing mobility analyzers, high-field asymmetric waveform ion mobility spectrometry (FAIMS) [29] should be mentioned. FAIMS represents a special separation technique that relies on the difference of ion mobilities in low and high electrical field (rather then on ion mobility itself). The definition of resolution for FAIMS devices is not wellestablished yet. Still, FAIMS can not be considered as a highly selective technique. The peak capacity of FAIMS spectra is typically well below 20 . Besides, the structural information on molecular cross-sections can not be easily extracted from FAIMS spectra.

For all known mobility analyzers, it is generally accepted that the random diffusion imposes a fundamental limit of the resolution. The ion propagation under the influence of electrical field constitutes a controlled motion; this process is disturbed by diffusional randomization. The only apparent way to improve the resolution is to increase the applied voltage, which certainly has a practical limit. Meanwhile, the way to over-pass this limit was already suggested more then a hundred years ago. In 1898, the so-called "parallel flow mobility analyzer" was proposed by J. Zeleny [30]. It is shown schematically in Figure 1. The sheath gas is blown through two grids. The voltage is applied 
to the grids, creating a uniform electrical field. The ions are generated between the grids. The electric field forces the ions to drift against the gas flow. The voltage is tuned to equilibrate the action of electrical force versus gas flow, for ions of interest. The ions of higher mobility move upstream, while the ions of lower mobility move downstream. Even if the difference in mobility is small, the ions can still be separated over an extended period of time. A very high-resolution is, theoretically, possible at any given flow rate and applied voltage. The parallel flow method of Zeleny demonstrates that a "simple" theory of the resolution needs to be modified. A more general theory, proposed by Zeleny [31], describes the diffusion-limited resolution as the ratio of the work of electrical field divided by ion thermal energy. For DT-IMS or DMA, the work of electrical force is proportional to the applied potential. For Zeleny's analyzer, the work is also proportional to the time the ions spend inside of the device. The residence time can be made arbitrarily long. Therefore, a high-resolution can be achieved even for modest voltages.

There are, however, several reasons that make the practical implementation of the parallel flow mobility analyzer problematic. The first problem is the difficulty with the injection of ions generated by any external ion source. Other problems have a more fundamental character. During a long residence time, necessary for ion separation, the ions undergo a random diffusion. The concentration of ions decreases, which makes the detection (or sampling) problematic. Finally, small variations in gas velocity or electrical field would break the precise balance between the electrical force and gas flow for ions of interest that is necessary for the device operation.

Zeleny did show the possibility to trade the resolution for longer separation time. The goal of the present work is the development of principally new mobility analyzer based on this principle, which would combine both high-resolution and high sensitivity with the convenience of interfacing to commercial mass spectrometers.

\section{Gas-Phase Ion Focusing: One-Dimensional Theory}

The proposed new principle of ion separation represents a modification of parallel flow analyzer (Figure 1) described above [30]. Let us start with one-dimensional case. Similar to parallel flow mobility device, ions move under the influence of electrical field and the buffer gas flow, where the two forces act in the opposite directions. However, the difference is that electrical field, buffer gas flow, or both are not uniform. For example, electrical field is increasing downstream, while the gas flow velocity remains permanent. Then, for the ions of interest with some value of mobility, there is equilibrium position where the action of gas flow is compensated by electrical force. If, due to the random diffusion, ions are shifted upstream (where the electrical field is weaker), they are turned back by a gas flow. If ions diffuse downstream (where the electrical field is stronger), then ions are moved back again. Therefore, ions of a certain kind are focused in a layer around the equilibrium position by combined action of uniform gas flow and electrical field (which is increasing downstream). The ions of different mobility are focused around another equilibrium position. Both (stationary in time) ion focusing and mobility separation are achieved. Another possibility to focus/separate the ions is to use a uniform electrical field combined with buffer gas flow that is decelerating downstream. Finally, the general case includes nonuniform electrical field and nonuniform gas flow. If, at some position, combined action of electrical field and gas flow move ions downstream and if, at another position downstream, the net force move ions upstream, there is an equilibrium position between, where the ions are focused/separated.

The one-dimensional focusing and separation of ions under the influence of nonuniform electrical field and gas flow, described above, allows for a simple analytical calculation. To begin with, let us calculate stationary in time ion density distribution $p(x)$ around the equilibrium position $x_{0}$, where $x$ is the coordinate. In the most general case, both the gas flow velocity and electrical field depend on the $x$-coordinate: $E=E(x)$ and $v=v(x)$. At the equilibrium position $x=0$ the electrical force is equilibrated by the gas flow: $v(0)=K E(0)$. Here $K$ is the ion mobility. For a small deviation of ions from equilibrium position $x=0$, a linear approximation for electrical field $E(x)$ and gas velocity $v(x)$ can be used:

$$
\begin{aligned}
E(x) & =E(0)+\frac{d E}{d x} \cdot x=E(0)+\beta x=\frac{v(0)}{K}+\beta x \quad v(x) \\
& =v(0)+\frac{d v}{d x} \cdot x=v(0)-a x
\end{aligned}
$$

In the eq $1, a$ is the gas flow acceleration, $\beta$ is the gradient of electrical field. I assume in the eq 1 that the gas velocity drops downstream, and the acceleration $a$ is positive. For stationary in time ion density distribution $p(x)$, the diffusional flow of ions $D \cdot d p(x) / d x$ (where $D$ is ionic diffusion coefficient) should be compensated by ion flow in opposite direction under the influence of gas flow and electrical field:

$$
\begin{aligned}
D & \cdot \frac{d \rho(x)}{d x} \rho \cdot[v(x)-K \cdot E(x)] \\
& =\rho \cdot\left[v-a x-K\left(\frac{v}{K}+\beta x\right)\right]=-\rho \cdot(a+K \beta) x
\end{aligned}
$$

Eq 2 can be easily integrated, giving the well-known Gaussian shape for ion density $\mathrm{p}(\mathrm{x})$ with half-width $\Delta x$ : 


$$
\begin{aligned}
\rho(x) & =\rho_{0} \cdot \exp \left[-\left(\frac{a}{2 D}+\frac{\beta \cdot e}{2 k T}\right) \cdot x^{2}\right] \\
& =\rho_{0} \cdot \exp \left[-\left(\frac{x}{\Delta x}\right)^{2}\right] \Delta x=\frac{1}{\sqrt{\frac{a}{2 D}+\frac{\beta \cdot e}{2 k T}}}
\end{aligned}
$$

To derive eq 3 from eq 2, the Nernst-TownsendEinstein relation between mobility $K$ and diffusion coefficient $D$ was used:

$$
K=e D / k T
$$

In eqs 3 and $4, k$ is the Boltzmann's constant; $e$ is the ion charge, and $T$ is the absolute gas temperature.

After the shape and half-width of the ion density function is calculated, it is possible to calculate the diffusion-limited resolution of the new technique. Let us discuss the focusing of ions with two slightly different ion mobilities, $K_{0}$ and $K_{1}$. The ions of both types are focused by nonuniform electrical field, and gas flow at different places, characterized by equilibrium coordinates $x_{0}$ and $x_{1}$ :

$$
v\left(x_{0}\right)-K_{0} E\left(x_{0}\right)=0 v\left(x_{1}\right)-K_{1} E\left(x_{1}\right)=0
$$

Both kinds of ions are focused in the two layers. The ion densities and layer thickness are determined by eq 3 . To be resolved, the centers of the two Gaussian distributions should be separated by double half-thickness $\Delta x$ :

$$
\left|x_{0}-x_{1}\right|=2 \Delta x=\frac{2}{\sqrt{\frac{a}{2 D}+\frac{\beta \cdot e}{2 k T}}}
$$

By definition, the resolution $R_{F}$ (determined by focusing), can be calculated as:

$$
R_{F}=K / \Delta K
$$

Using eq 5 and linear approximation for electrical field and gas flow velocity (eq 1), we can calculate $\Delta K$ as:

$$
\Delta K=\frac{v \beta+E a}{E^{2}} \cdot\left|x_{1}-x_{0}\right|
$$

Eq 8 was calculated using the assumption that the variations of both electrical field and gas flow velocity for close points $x_{0}$ and $x_{1}$ are small, compared to the corresponding values of electrical field and velocity themselves: $E\left(x_{0}\right) \approx E\left(x_{1}\right)=\mathrm{E} ; v\left(x_{0}\right) \approx v\left(x_{1}\right)=v$. The same is valid for ion mobility: $K_{0} \approx K_{1}=v / E$. Now we substitute eq 6 into 8 :

$$
\begin{aligned}
\Delta K & =\left(\frac{K \beta}{E}+\frac{a}{E}\right) \cdot \frac{2}{\sqrt{\frac{a}{2 D}+\frac{\beta \cdot e}{2 k T}}} \\
& =K \cdot\left(\frac{\beta}{E}+\frac{a}{K E}\right) \cdot \frac{2}{\sqrt{\frac{a}{2 D}+\frac{\beta \cdot e}{2 k T}}}
\end{aligned}
$$

Finally, the resolution $R_{F}$ can be derived as follows:

$$
R_{F}=\frac{K}{\Delta K}=\frac{\sqrt{\frac{a}{2 D}+\frac{\beta \cdot e}{2 k T}}}{2 \cdot\left(\frac{\beta}{E}+\frac{a}{K E}\right)}=\frac{K E \cdot \sqrt{\frac{a}{2 D}+\frac{\beta \cdot e}{2 k T}}}{2(K \beta+a)}
$$

Eq 10 provides the analytical expression for diffusion-limited resolution of ions, focused and separated by arbitrary one-dimensional gas flow with acceleration $a$ and electrical field gradient $\beta$.

Eqs 3 and 10 demonstrate that both ion focusing and mobility separation can be achieved using nonuniform electrical field and gas flow. I have called the new type of mobility separation, which is based on the principle described above, focusing ion mobility spectrometry. Let us estimate the diffusion-limited resolution of focusing IMS for different experimental conditions. The first example is the case with constant gas flow velocity and electrical field increasing downstream: gas acceleration $a=0$; electrical field gradient $\beta \neq 0$. Eqs 2 and 10 can now be simplified:

$$
\Delta x=\sqrt{\frac{2 k T}{\beta \cdot e}} \quad R_{F}=E \cdot \sqrt{\frac{e}{8 k T \cdot \beta}}
$$

To estimate the order of magnitude for ion layer thickness $\Delta x$ and diffusion-limited resolution $R_{F}$, the following set of parameters is used: $E=200 \mathrm{~V} / \mathrm{cm} ; \beta=$ $d E / d x=1 \mathrm{~V} / \mathrm{cm}^{2} ; k=1.38 \cdot 10^{-23} \mathrm{~J} / \mathrm{grad} ; \mathrm{T}=300{ }^{\circ} \mathrm{K} ; e=$ $1.6 \cdot 10^{-19} \mathrm{~K}$ (for singly-charged ions). The calculation gives $\Delta x=0.23 \mathrm{~cm}$ and $R_{F}=440$. To equilibrate the electrical field of $200 \mathrm{~V} / \mathrm{cm}$, a reasonable buffer gas velocity of $100 \mathrm{~cm} / \mathrm{s}$ is to be maintained if the ions of interest have a "typical" mobility of $0.5 \mathrm{~cm}^{2} / \mathrm{Vs}$.

The second important case is ion focusing/separation in uniform electrical field $(\beta=0)$ and decelerating gas flow $(a \neq 0)$. Eqs 2 and 10 can be rewritten as:

$$
\Delta x=\sqrt{\frac{2 D}{a}} \quad R_{F}=\frac{v}{\sqrt{8 D a}}=E \cdot \sqrt{\frac{e K}{8 a k T}}
$$

The calculation for singly charged ions with the following set of parameters: $v=200 \mathrm{~cm} / \mathrm{s} ; a=1 \mathrm{~cm} / \mathrm{s}^{2}$; $K=0.5 \mathrm{~cm}^{2} / \mathrm{Vs}$, gives the values of layer thickness $\Delta x$ $=1.6 \mathrm{~mm}$ and the resolution $R_{F}=620$. Theoretically, unlimited resolution $R_{F}$ can be achieved even with 


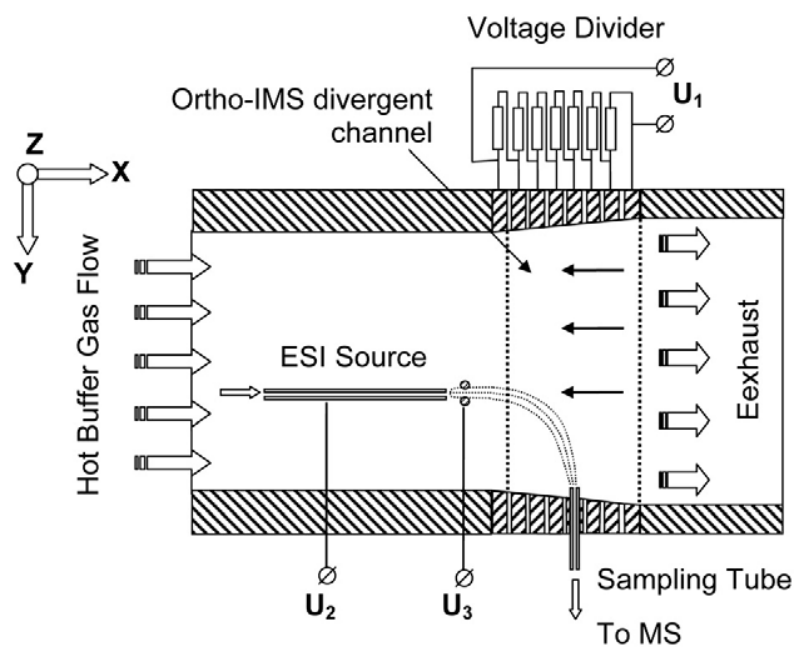

Figure 2. Schematic view of orthogonal extraction IMS.

moderate values of electrical field and/or flow velocity if the gradient of electrical field (eq 11) or flow acceleration (eq 12) is small enough.

\section{Orthogonal IMS: Instrument Design}

The principle of focusing IMS for simplified onedimensional model, described in the previous section, can be implemented in a number of different experimental designs. Some of them can use a buffer gas flow with uniform velocity; others can be based on more general arbitrary flow and electrical field profiles. Gas-transparent grids of various shapes can be used in focusing IMS designs to establish the desirable field and flow profiles. Gridless designs are also a possibility. More detailed discussion of different possible schemes will be presented elsewhere. In the present publication, I focus entirely on one particular implementation, which is adopted for efficient interfacing to MS instruments.

Focusing IMS device, described below, is based on the ion focusing/separation in a uniform electrical field and decelerating buffer gas flow. It is called orthogonal extraction IMS or ortho-IMS. The implementation of ortho-IMS, shown in Figure 2, is equipped with electrospray source. A stream of the buffer gas flows through the ion source chamber. The buffer gas (an ambient air can be used) is supplied by appropriate air pump (not shown). ESI ion source is installed inside of the source chamber in the vicinity to the inlet grid. The voltage $U_{2}$ is applied to electrospray the liquid. The additional focusing ring electrode can be (optionally) installed between the spray tip and the inlet grid. Focusing ring concentrates the stream of electrosprayed ions in a relatively narrow beam. The voltage $U_{3}$ is applied to the ring electrode. With the flow of buffer gas (that can be heated to assist the electrospray ionization) the beam of ions enters the inner ortho-IMS channel through its inlet grid. The channel consists of several metal guard frames that are electrically isolated from each other, and from the inlet and outlet metal grids. The uniform electrical field $E$ is established inside the channel through the application of the voltage $U_{1}$ to the grids and to the guard frames (through the appropriate voltage divider). Another important function of inlet/outlet grids is to establish a plug flow profile inside the inner ortho-IMS channel. The potential $U_{1}$ can be tuned (or slowly scanned for the recording of IMS spectrum) depending on gas velocity and mobility of ions to be sampled. The ion motion inside the channel slows down because the electrical force acts in the direction opposite to the flow. The channel is profiled in such a way that its cross-section is gradually increasing downstream. Therefore, the gas velocity drops downstream. Under the combined influence of the uniform electrical field and divergent/ decelerating gas flow, the beam of ions of interest slows down inside and cannot move deeper behind some equilibrium surface. The character of ion motion in "horizontal" $x y$-plane and in "vertical" $x z$ plane is different and needs to be discussed separately. In "horizontal" cross-section, the inner orthoIMS channel is divergent (Figure 3a). Gas flow in this plane has $y$-components directed toward the channel walls. In $x$-direction, ion motion is slowed down by the electrical field. As a result, ions move in $y$ direction (orthogonally to their initial $x$-direction) toward the inlet orifice of the sampling capillary interfaced to a mass spectrometer. The sampling (or extraction) of ions in the direction orthogonal to ion injection is essential to ortho-IMS operation and provides the justification for its name. Ions with higher mobility are either discharged on the guard frame downstream of the capillary, or passed through the exit grid. Ions with lower mobility are discharged either on the guard frame upstream of the capillary, or on the inlet grid. The sampling capillary is inserted through the hole in the guard frame approximately in the middle of the inner channel and is adapted to transfer the ions to the inlet of MS instrument. The flow into the mass spectrometer is low (typically 1-2 $1 / \mathrm{min}$ ), and therefore, the sampling does not disturb the gas flow profile inside ortho-IMS essentially.

In the "vertical" cross-section (Figure 3b), the channel is profiled to be slightly convergent $\left(z_{\text {in }}>z_{\text {out }}\right.$, Figure $3 b$ ). To ensure that the gas flow decelerates downstream, (which is a condition for ion focusing/ separation), the area of inlet grid should be lower then the area of outlet grid: $y_{\text {in }} \times z_{\text {in }}<y_{\text {out }} \times z_{\text {out }}$. Gas flow in $x z$-plane has velocities with $z$-components directed toward the middle channel $x z$ cross-section. The electrical field has no $z$-components; thus, the beam of ions of interest is concentrated toward the middle instrument $x y$-plane by a convergent gas flow. The convergence of inner ortho-IMS channel in $z$-direction suppresses the diffusion of slowly moving ion beam in this direction. In other words, ortho-IMS design provides both sepa- 

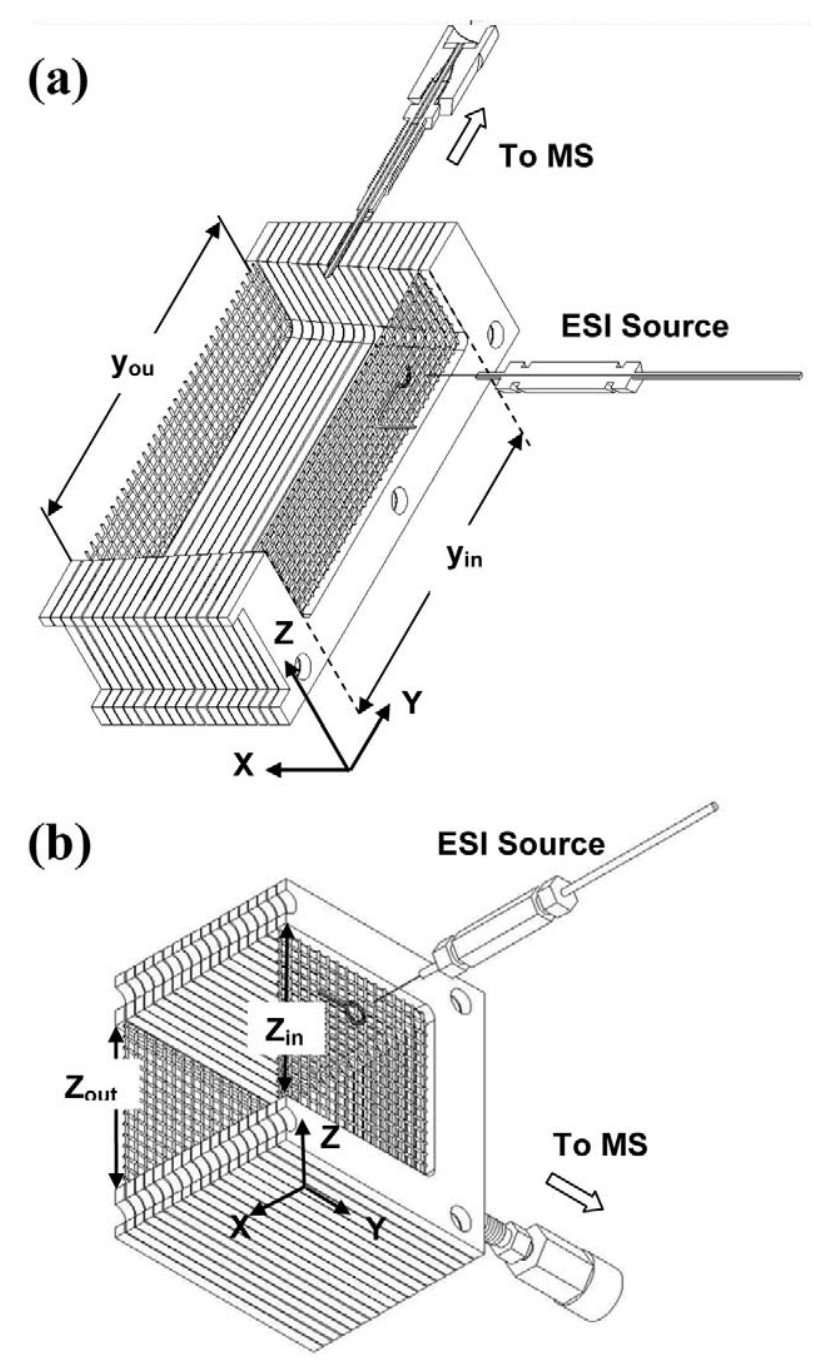

Figure 3. Middle cross-sections of ortho-IMS. (a) Middle $x y$ cross-section of ortho-IMS. The analyzer channel is divergent in $x$-direction: $y_{\text {in }}<y_{\text {out }}$. (b) Middle $x z$ cross-section of ortho-IMS. The analyzer channel is convergent in $x$-direction: $z_{\text {in }}>z_{\text {out }}$.

ration and three-dimensional focusing of ions necessary for efficient sampling to MS instrument.

The operation of ortho-IMS, qualitatively described in this section, hardly allows for analytical calculation. To calculate the resolution and sampling efficiency of the device, numerical computer simulation of ion motion was performed, and is described in the following section.

\section{Orthogonal IMS: Numerical Simulation}

Special software was developed in-house to simulate the tree-dimensional ion motion inside ortho-IMS. A model with uniform electrical field and plug gas flow profile were used for the calculations. Tree-dimensional trajectory of each ion was calculated. Ion diffusion was simulated using the Monte-Carlo method. The displacement $\Delta l$ of ion at fixed short time interval $\Delta t$ was calculated as a sum of displacements $\Delta l_{1}$ (due to the influence of electrical field and gas flow) and $\Delta l_{2}$ (in randomly selected direction due to diffusion effect). The diffusion constant $D$ is related to $\Delta t$ and $\Delta l$ in the following way [32]: $D=(\Delta l)^{2} / 4 \Delta t$. The ortho-IMS geometry/flow/voltage parameters, described in the previous section, were used for the calculations: inlet grid size $8 \times 4 \mathrm{~cm}$; outlet grid $8.2 \times 3.95 \mathrm{~cm}$; channel length $4 \mathrm{~cm}$; flow velocity $2 \mathrm{~m} / \mathrm{s}$; voltage of $1586 \mathrm{~V}$. Figure 4a shows calculated ion trajectories for two ions with mobility of 0.5000 and $0.5006 \mathrm{~cm}^{2} / \mathrm{Vs}$ (the difference is only $0.15 \%$ ). This example demonstrates the final ion spatial separation of several millimeters in $x y$-plane.

\section{Resolution}

Trajectories of 10,000 ions (half of them with mobility of 0.5000 and another half of $0.5006 \mathrm{~cm}^{2} / \mathrm{Vs}$ ) were started randomly within a circle of $4 \mathrm{~mm}$ diameter that corresponds to the plume size of a typical nano-electrospray source. The center of the circle was shifted off-axes in $y$-direction by $4 \mathrm{~mm}$ to direct an ion beam toward the sampling orifice. In Figure $4 \mathrm{~b}$ the two histograms of final $x$-coordinates for both types of ions are shown. As it can be seen from the picture, spatial separation is achievable for ions with difference in mobilities of just $0.15 \%$, which corresponds to the diffusion-limited resolution of more then 660 .

\section{Ion focusing}

Figure $4 \mathrm{c}$ demonstrates the histograms of final $\mathrm{z}$ coordinates for 10,000 ions (both kinds). In spite of the diffusion, the ion beam with initial $z$-coordinate spread of $4 \mathrm{~mm}$ is compressed by the convergent gas flow and focused with spread of $\approx 2 \mathrm{~mm}$ (more then $60 \%$ ), that can be efficiently sampled to MS inlet capillary (Figures 2 and 3).

\section{Separation time}

The distribution of ion time-of-flights were calculated as well (data not shown). Ion time-of-flights statistically vary in the range of $1.44-3.3 \mathrm{~s}$. The long separation time is the price that was paid for high-resolution. In DTIMS, stronger electrical field works for a shorter time (of the order of several milliseconds). In ortho-IMS, the action of weaker field is used for a much longer time.

\section{Discussions}

The suggested orthogonal extraction IMS has two important advantages over both drift-tube IMS and differential mobility analyzer: much higher diffusion-limited resolution and efficient ion transmission. It works as a mobility filter, passing only ions with a narrow mobility window. To record the whole IMS spectrum, this slow scanning is necessary. In this respect, it is similar to DMA. In DMA, ions pass two narrow slits which results in essential losses. In the suggested ortho-IMS design, 
(a)
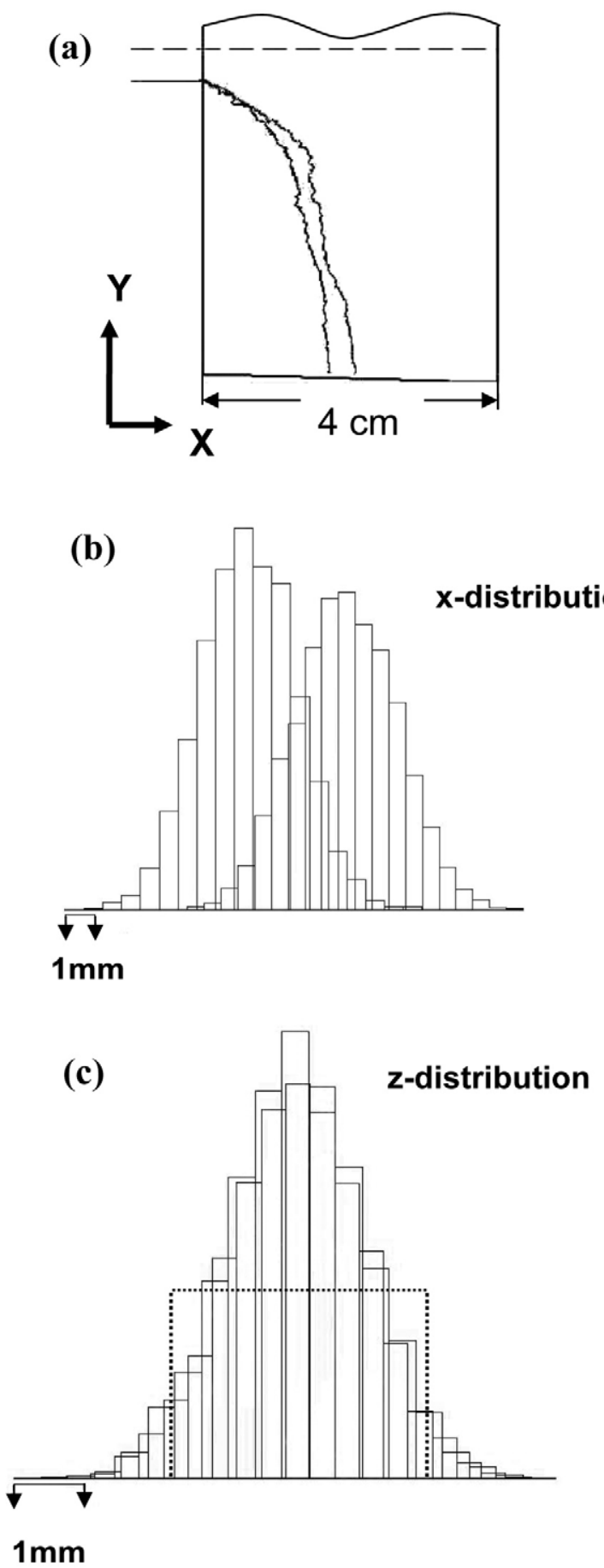

Figure 4. Three-dimensional Monte-Carlo numerical simulation of ortho-IMS operation. (a) Calculated ion trajectories inside of ortho-IMS channel ( $x y$-plane). The two traces correspond to ions with mobilities $K=0.5000$ and $0.5008 \mathrm{~cm}^{2} / \mathrm{Vs}$ (difference $0.15 \%$ ). The irregularities in trajectories are the consequence of ion diffusion simulated with statistical Monte-Carlo calculation. (b) The calculated $x$-distributions of ion final ion position. The two distributions differ by $0.15 \%$ of ion mobility, which corresponds to the resolution of 660 . The trajectories of 5000 ion of every kind were calculated. (c) The calculated $z$-distributions of ion final ion position. The initial random ion $z$-coordinate distribution with the width of $4 \mathrm{~mm}$ is shown with dashed line. ions (on their way to mass spectrometer) pass only one grid and are additionally 3D focused toward MS entrance. Therefore, ion transmission of ortho-IMS-MS combined instruments is expected to be high and independent on the resolution.

Ortho-MS is a much slower spectrometer compared with DT-IMS and even with DMA. Ions need to spend a long time inside its chamber before sampling. The higher the resolution is targeted, the longer is the time. High-resolution ion mobility spectrum can be recorded by slow scanning of applied voltage. This is the price for the ortho-IMS resolution. On the other hand, such a device can be easily adapted to fast or slow MS instruments of different types.

Another attractive feature of ortho-IMS is that the ion focusing and separation process is "self-adjustable". Small (but not dependent on time) deviations of electrical field and/or gas flow profiles would not break its operation; ions would just be focused at a slightly different place. The phenomenon can be easily compensated. Of course, any flow pulsation/turbulence kills the resolution of ortho-IMS. High-quality gas pumps, big dumping vessels, additional grids could be used to eliminate the undesirable flow pulsations. Another possibility is to use ortho-IMS of bigger size and slower gas flow velocity.

The sampling capillary disturbs the gas flow profile in its proximity as well. This short-range disturbance (the sampling flow is assumed to be much less than the main gas flow) takes place in the region where ions are already separated and will have a minor effect on the resolution.

A buildup of space charge is another possible resolution-limiting factor. Ions spend a rather long time inside of ortho-IMS before they are sampled or detected; therefore, increased volume ion density could disturb the uniformity of the electrical field. Still, this problem may not be too limiting for two reasons. Only a small part of the variety of generated ions spends essential time inside the ortho-IMS channel. Ions of too high mobility are discharged at the inlet grid of the instrument; ions of too low mobility pass through relatively quickly. Only a (small) portion of ions will contribute to the space charge. Second, a small deviation of electrical field would just slightly change the ion trajectories, while these ions would still be focused.

\section{Conclusions}

A new principle of gas-phase separation of ions based on their mobility is suggested. Focusing IMS uses ion focusing/separation under combined action of electrostatic field and gas flow; gas flow velocity, electrical field, or both are not uniform. The analytical calculation of one-dimensional model, based on the first principles, demonstrates that a diffusion-limited resolution of several hundred is achievable at moderate values of voltages and flow rates. One possible implementation of the 
focusing IMS principle, which is called orthogonal IMS, is discussed in detail. A convergent in one direction and divergent in another direction gas flow focuses the ion beam, providing the efficient sampling of the ions to mass spectrometer in the direction orthogonal to the gas flow. Three-dimensional Monte-Carlo numerical computer simulation of ion motion predicts a diffusionlimited resolution of ortho-IMS higher then 600 and efficient ion focusing toward its sampling capillary.

\section{Acknowledgments}

The author has conducted the present research, having been inspired by the development of the ortho-TOF technique by Alexander Dodonov-his teacher in the field of mass spectrometry and far beyond. It was to have been the author's homework from him. Unfortunately, he was not able to complete it in time. Prof. Dodonov passed away on August 31, 2005. The author dedicates this article to his memory.

\section{References}

1. Cohen, M. J.; Karasek, F. W. Plasma Chromatography-A New Dimension for Gas Chromatography and Mass Spectrometry. J. Chromatogr. Sci. 1970, 8, 330 .

2. St. Louis, R. H.; Hill, H. H. Ion Mobility Spectrometry in Analytical Chemistry. Crit. Rev. Anal. Chem. 1990, 21, 330

3. Hill, H. H.; Siems, W. F.; St. Louis, R. H.; McMinn, D. G. Ion Mobility Spectrometry. Anal. Chem. 1990, 62, 1201A.

4. Cottingham, K. Ion Mobility Spectrometry Rediscovered. Anal. Chem. 2003, 75, 435A-439A.

5. Fenn, J. B.; Mann, M.; Meng, C. K.; Wong, S. F.; Whitehouse, C. M. Electrospray Ionization-Principles and Practice. Mass Spectrom. Rev. 1990, 9, 37.

6. Tanaka, M.; Ido, Y.; Akita, S.; Yoshiba, T. Protein and Polymer Analysis up to $m / z 100,000$ by Laser Ionization Time-of-Flight Mass Spectrometry. Rapid Commun. Mass Spectrom. 1988, 2, 151-153.

7. Karas, M.; Hillenkamp, F. Laser Desorption Ionization of Proteins with Molecular Masses Exceeding 10,000 Daltons. Anal. Chem. 1988, 60, 2299-2301.

8. Gieniec, J.; Mack, L. L.; Nakamae, K.; Gupta, C.; Kumar, V.; Dole, M Electrospray Mass-Spectroscopy of Macromolecules-Application of an Ion-Drift Spectrometer. Biomed. Mass Spectrom. 1984, 11(6), 259-268.

9. Shumate, C. B.; Hill, H. H. Coronaspray Nebulization and Ionization of Liquid Samples for Ion Mobility Spectrometry. Anal. Chem. 1989, 61, 601.

10. Smith, R. D.; Loo, R. R.; Busman, M.; Udseth, H. R. Principles and Practice of Electrospray Ionization-Mass Spectrometry for Large Polypeptides and Proteins. Mass Spectrom. Rev. 1991, 10, 359-451.

11. Wittmer, D.; Chen, Y. H.; Luckenbill, B. K.; Herbert, H. H. Electrospray Ionization Ion Mobility Spectrometry. Anal. Chem. 1994, 66, 2348-2355.
12. Hoaglund-Hyzer, C. S.; Counterman, A. E.; Clemmer, D. E. Anhydrous Protein Ions. Chem. Rev. 1999, 993037.

13. Colgrave, M. L.; Bramwell, C. J.; Creaser, C. S. Nanoelectrospray Ion mobility Spectrometry and Ion Trap Mass Spectrometry Studies of the Noncovalent Complexes of Amino Acids and Peptides with Polyethers. Int. J. Mass Spectrom. 2003, 229, 209-216.

14. Woods, A. S.; Fuhrer, K.; Gonin, M.; Egan, T.; Ugarov, M.; Gillig, K. J.; Schultz, J. A. J. Angiotensin II-Acetylcholine Noncovalent Complexes Analyzed with MALDI-Ion Mobility-TOF MS. Biomol. Tech. 2003, 14, $1-8$.

15. Ruotolo, B. T.; Verbeck, G. F.; Thomson, L. M.; Woods, A. S.; Gillig, K. J.; Russell, D. H. Distinguishing Between Phosphorylated and Nonphosphorylated Peptides with Ion Mobility-Mass Spectrometry. J. Proteome Res. 2002, 1, 303-306.

16. Kindy, J. M.; Taraszka, J. A.; Regnier, F. E.; Clemmer, D. E. Quantifying Peptides in Isotopically Labeled Protease Digests by Ion Mobility/ Time-of-Flight Mass Spectrometry. Anal. Chem. 2002, 74, 950-958.

17. Barnes, C. A. Srebalus; Hilderbrand, A. E.; Valentine, S. J.; Clemmer, D. E. Resolving Isomeric Peptide Mixtures: A Combined HPLC/Ion Mobility-TOFMS Analysis of a 4000-Component Combinatorial Library. Anal. Chem. 2002, 74, 26-36.

18. Baker, E. S.; Bernstain, S. L.; Bowers, M. T. Structural Characterization of G-Quadruplexes in DeoxyGuanosine Clusters Using Ion Mobility Mass Spectrometry. J. Am. Soc. Mass Spectrom. 2005, 16, 989-997.

19. Loo, J. A.; Berhane, B.; Kaddis, C. S.; Wooding, K. M.; Xie, Y.; Kaufman, S. L.; Chernushevich, I. V. Electrospray Ionization Mass Spectrometry and Ion Mobility Analysis of the 20S Proteasome Complex. J. Am. Soc. Mass Spectrom. 2005, 16, 998-1008.

20. Mamyrin, B. A.; Karataev, D. V.; Schmikk, D. V.; Zagulin, V. A.MassReflectron, a New Nonmagnetic Time-of-Flight High-Resolution Mass Spectrometer. Sov. Phys. JETP 1973, 37, 45

21. Dodonov," A. F., Chernushevich," I. V., Dodonova,, T. F., Raznikov," V. V., Tal'rose,", V. L. Inventor's Certificate No. 1681340A1, USSR, February 25, 1987.

22. Dodonov , A. F., Chernushevich, I. V., Laiko, V. V. Time-of-Flight Mass Spectrometry. 1994, ACS Symposium Series 549, Chap. VII.

23. St. Louis, R. H.; Hill, H. H., Jr. Ion Mobility Spectrometry in Analytical Chemistry. Crit. Rev. Anal. Chem. 1990, 21, 321-355.

24. Knorr, F. J.; Eatherton, R. L.; Siems, W. F., Hill, H. H. Fourier Transform Ion Mobility Spectrometry. Anal. Chem. 1985, 57, 402.

25. Clowers, B. H.; Massick, S.; Siems, W. F.; Hill, H. H.Hadamard Transform Electrospray Ionization Atmospheric Pressure Ion Mobility Spectrometry. Abstracts; Proceedings of the 53rd Annual Conference on MS and Allied Topics San Antonio TX, June 2005 p 187.

26. Kaufman, S. L.; Skogen, J. W.; Dorman, F. D.; Zarrin, F.; Lewis, K. C. Macromolecule Analysis Based on Electrophoretic Mobility in Air: Globular Proteins. Anal. Chem. 1996, 68, 1895-1904.

27. Flagan, R. C. History of Electrical Aerosol Measurements. Aerosol Sci. Technol. 1998, 29, 301-380.

28. De la Mora, J. F.; Thomson, B. A.; Gamero-Castano, M. Tandem Mobility Mass Spectrometry Study of Electrosprayed Tetraheptyl Ammonium Bromide Clusters. J. Am. Soc. Mass Spectrom. 2005, 16, 717-732.

29. Purves, R. W.; Guevremont, R.; Day, S.; Pipich, C. W.; Matyjaszczyk, M. S. Mass Spectrometric Characterization of a High-Field Asymmetric Waveform Ion Mobility Spectrometer. Rev. Sci. Instrum. 1998, 69, 4094-4105.

30. Zeleny, J. Mobilities of the Ions in Gases at Low Pressures. Philos. Mag. 1898, 46, 120-154.

31. Zeleny, J. The Distribution of Mobilities of Ions in Moist Air. Phys. Rev. 1929, 34, 310-334.

32. Berry, R. S.; Rice, S. A., Ross, J. Physical Chemistry; Wiley, N.Y.: 1980; pp 142-143. 